\title{
TAMAÑO DE FAMILIA Y ORDEN DE NACIMIENTO EN CHILE: USANDO GEMELOS COMO EXPERIMENTO NATURAL
}

\author{
FAMILY SIZE AND BIRTH ORDER IN CHILE: USING TWINS AS A \\ NATURAL EXPERIMENT
}

\section{CLAUDIA SANHUEZA* \\ ILADES/Universidad Alberto Hurtado}

\begin{abstract}
This study estimates the causal effect of family size and birth order on educational attainment of individuals in the long run. Following recent literature we use the presence of twins as an instrumental variable for family size and fixed effects model for birth order. The results suggest that in Chile there is a negative relationship between family size and individuals' educational achievements. Moreover, it shows a strong significant and positive effect of being a big brother. However, in larger families there is a nonlinear effect in which middle siblings are less favored.
\end{abstract}

Keywords: Birth order, family size, family fixed effects, twins, natural experiments.

JEL Classification: J12, J13.

\section{Resumen}

Esta investigación estima el efecto causal del tamaño de la familia y el orden de nacimiento sobre los logros educacionales de los individuos en el largo plazo. Siguiendo la literatura reciente usamos la presencia de gemelos como variable instrumental para el tamaño de la familia y modelo de

* Email: csanhuez@uahurtado.cl. Agradezco sinceramente a Claudio Fuentealba por su ayuda en una primera versión de este estudio, así como a los dos anónimos referees de la Revista de Análisis Económico ILADES/Georgetown University. 
efectos fijos para el orden de nacimiento. Los resultados sugieren que en Chile existe una relación negativa entre el tamaño de la familia y los logros de los individuos en hogares. Por otra parte, se muestra un fuerte efecto significativo y positivo de ser el hermano mayor. Sin embargo, en familias más numerosas se observa un efecto no lineal siendo menos favorecidos los hermanos del medio.

Palabras Clave: Orden de nacimiento, tamaño de familia, efectos fijos de familia, gemelos, experimento natural.

Clasificación JEL: J12, J13.

\section{INTRODUCCION}

El estudio de la influencia de la familia sobre el desarrollo de los individuos y su éxito socioeconómico durante la adultez es de gran relevancia en la investigación, no sólo económica, sino también en variadas ramas de las ciencias sociales como la psicología y sociología. La calidad de la niñez, determinada entre otros factores por el tiempo que los padres pasan con los hijos, así como la interacción de los menores con sus hermanos y el efecto del entorno familiar en general sobre el logro futuro de los hijos ha sido ampliamente desarrollado teóricamente. Este desarrollo se puede distinguir entre los tempranos postulados de Becker (Becker, 1960; Becker y Lewis, 1973; Becker y Tomes, 1976) que plantea la idea de un trade-off entre la calidad y cantidad de hijos y los planteamientos más recientes que postulan la posible existencia de externalidades positivas en la interacción entre los hermanos (Behrman, Pollack y Taubman, 1995).

Sin embargo, el desarrollo de investigaciones empíricas que determinen el efecto de distintos aspectos de la estructura familiar sobre los logros futuros de los individuos ha ido avanzando con un cierto rezago que responde no a la falta de interés, sino más bien a la falta de datos que permitan desarrollarlas. Este rezago es especialmente importante en países en desarrollo como el caso de Chile.

Rompiendo con esto, en esta investigación se utiliza la Encuesta de Protección Social 2002 que pone a disposición de los investigadores, por primera vez para Chile, información que permite la investigación de efectos del tamaño de la familia y el orden de nacimiento sobre los logros subsecuentes de los individuos. Esta encuesta posee preguntas sobre la historia familiar e individual de los entrevistados, como todos los menores con los cuales vivió, la educación y la edad, la educación y edad de los padres y el nivel socioeconómico del hogar en la infancia.

Uno de los principales problemas de identificación del efecto del tamaño de la familia sobre la educación es que el tamaño del hogar se determina endógenamente. Por lo tanto, la literatura reciente en países desarrollados enfrenta esto utilizando variaciones exógenas al tamaño del hogar. Así, Conley y Glauber (2005) para Estados Unidos utilizan el sexo de los dos primeros hijos y Black, Devereux y Salvanes (2005) para Noruega utilizan la presencia de gemelos. 
Siguiendo la literatura reciente nosotros enfrentamos el tema de la endogeneidad del tamaño del hogar usando la existencia de gemelos como variable instrumental para el tamaño de la familia. Los resultados sugieren la existencia en Chile de una relación negativa entre el tamaño de la familia y los logros de los individuos, según la cual personas que provienen de familias más numerosas logran en promedio menores años de estudio. Además, se encuentra un efecto no lineal del orden de nacimiento, según el cual se ven favorecidos claramente los individuos nacidos primeros en todo tipo de familias, y también los últimos en familias más numerosas.

En la sección II se resumen las teorías que hacen esperar efectos del tamaño de la familia, el orden de nacimiento y el espacio temporal que separa el nacimiento de los hermanos sobre los logros individuales. La sección III describe la muestra y además presenta una primera indagación en los datos. Finalmente, las secciones IV y V presentan los resultados en tamaño de la familia y orden de nacimiento respectivamente para finalizar con las conclusiones en la sección VI.

\section{REVISION DE LA LITERATURA}

\subsection{El tamaño de la familia}

En general las hipótesis sobre los efectos del tamaño de la familia predicen que aquellos individuos que provienen de familias más numerosas obtendrán peores resultados en relación a individuos de familias más pequeñas ya que, ceteris paribus, tendrán a su disposición una menor cantidad de recursos. En este sentido, operan conceptos netamente económicos como la rivalidad entre hermanos que busca iluminar la noción de que más hermanos (consumidores) competirán por recursos escasos. $\mathrm{O}$ bien el postulado de Becker (Becker, 1960; Becker y Lewis, 1973; Becker y Tomes, 1976) quien trabaja en un sentido muy semejante la existencia de un trade-off entre calidad y cantidad de los niños en la medida que la familia crece. Cabe destacar que un rol muy importante en estas hipótesis se le da a la existencia de una restricción presupuestaria. Cuando hablamos de recursos cuya dotación es fija, como por ejemplo el tiempo de los padres, la rivalidad entre hermanos opera sin plantear la necesidad de hacer más supuestos o de acotar la situación en que se esperan sus efectos. Sin embargo, la inversión en alimentación, salud, recreación, etc. dependerá también de factores de mercado. Como se señala en Bauer y Gang (2001) cuando la restricción presupuestaria no es restrictiva, la inversión en educación en cada hijo debería realizarse hasta un punto en que el retorno marginal de ésta iguale el costo de obtener los fondos, es decir, la tasa de interés, de tal forma que esta inversión sería independiente del tamaño de la familia. Sin embargo, cuando ésta es restrictiva (por imperfecciones del mercado de capitales, por ejemplo) la forma de maximizar el retorno monetario de los recursos financieros disponibles sería invirtiendo más en aquellos individuos que presentan un mayor retorno. Lo que hace que el tamaño de la familia comience a importar. 
Pero no sólo desde la economía han surgido hipótesis al respecto. Desde la psicología destaca el modelo de confluencia (Zajonc, 1976) el cual señala que es la madurez promedio de la familia en que crece el individuo el factor determinante en sus logros. En la medida que la madurez está fuertemente relacionada con la edad, la edad promedio de la familia en que crece el niño tendrá efectos sobres sus logros. Así, en la medida que cada nuevo hijo contribuye a diluir la madurez entre más personas (lo que se refleja en que disminuye la edad promedio de la familia) familias más numerosas tendrían un peor desempeño en términos relativos.

La evidencia empírica, sin embargo, se encuentra aún en construcción. Steelman et al. (2002) hacen una compilación de los resultados reportados de la relación entre tamaño de la familia y logros educacionales, en la cual se observa una relación negativa altamente estable. Se señala que al menos para Estados Unidos y Europa del Este la evidencia al respecto ha sido inequívoca. Sin embargo, hay resultados que cuestionan este patrón. Kessler (1991) utilizando datos para Estados Unidos no encuentra evidencia significativa del efecto del tamaño de la familia sobre el nivel de salarios del individuo ni tampoco sobre su crecimiento. También Wells (1995) no puede rechazar la hipótesis de que el efecto del tamaño de la familia sobre los logros educacionales es estadísticamente distinto de cero. El autor simplemente declara que no encuentra una explicación para no haber podido replicar un resultado largamente documentado. Más tarde Bauer y Gang (2001) con datos de Alemania tampoco logran replicar este resultado, pero ellos sí le dan una interpretación a ese hecho, señalando que la falta generalizada de significancia encontrada en él se debería a que en Alemania la restricción presupuestaria no es restrictiva en lo que respecta a la asistencia al colegio (algo ciertamente verosímil dado el carácter de la educación pública del país en cuestión).

Sin embargo, hay desafíos metodológicos pendientes. La posibilidad de que estos hallazgos sean resultado de la influencia de otros factores no medibles correlacionados con el tamaño del hogar o la endogeneidad del tamaño del hogar, haciendo necesaria la búsqueda de variaciones exógenas. Guo y Van Wey (1999) tras un riguroso testeo de la hipótesis de la dilución de recursos, usando un análisis de datos longitudinales, destacando el carácter dinámico de la relación entre el tamaño de la familia y los logros educacionales, no encuentran efectos significativamente distintos de cero. Conley y Glauber (2005), utilizando como variable instrumental la composición de género del grupo de hermanos, encontraron que familias con dos hijos de igual sexo tiene un $7 \%$ más de probabilidades de tener un tercer hijo en comparación con familias que tienen un par mixto de hijos. Toda vez que el sexo de los hijos es una variable aleatoria, se tiene una variación en la propensión a tener un tercer hijo que no se asocia a ningún otro factor familiar. Sus resultados son menores que los encontrados típicamente pero distintos de cero. Refiriéndose explícitamente al trabajo de Guo y Van Wey (1999), la discusión metodológica y la tarea de establecer el impacto último del tamaño de la familia sobre los logros educacionales están lejos de estar resueltas. Más recientemente Black, Devereux y Salvanes (2005) utilizando datos administrativos para Noruega encuentran un efecto no significativo del tamaño del hogar. Estos usan como variable instrumental para el tamaño del hogar la presencia de gemelos los que aumentan el tamaño del hogar exógenamente a sus características. 


\subsection{El orden de nacimiento}

En general las hipótesis con respecto al efecto del orden de nacimiento sobre los logros futuros de los hijos se contradicen y existe menos acuerdo con respecto a la validez de cada una de ellas.

Desde la biología genética surgen dos argumentos a favor del desempeño relativo de los niños que nacen primero. La probabilidad de nacimientos con problemas genéticos aumenta en la medida que la madre tiene más edad, en consecuencia los hijos que nacen más tarde tienen más probabilidad de nacer con un peor acervo genético. La probabilidad de nacimientos bicigóticos (gemelos, mellizos o siameses) también está positivamente correlacionada con la edad, en la medida que éstos competirán por recursos durante su gestación así como durante su infancia (ya que el tamaño de la familia es mayor); niños de nacimiento más tardío tenderían a tener peores logros futuros. Por otra parte, la imposibilidad de conocer el acervo genético de los hijos que están por nacer también haría que se invierta más en los hijos que nacen primero, ya que hay menos grados de libertad al evaluar el rendimiento de esta inversión (Behrman y Taubman, 1986; Behrman, Pollack y Taubman, 1995).

No obstante, el modelo psicológico de Zajonc (1976) también se puede articular para dar un argumento en esta parte de la discusión. En la medida que el logro de los hijos depende de la madurez promedio de la familia en la que crecen, y ésta disminuye en la medida que nacen más niños, quienes nacen primero se ven beneficiados de un ambiente más maduro durante los años en que viven sin hermanos menores. Por otra parte, el mismo autor hace una excepción que permitirá introducirnos en una segunda línea de argumentos. Zajonc (1976) habla acerca del efecto profesor refiriéndose a los beneficios que trae para el hermano mayor el rol que jugaría enseñando cosas a los hermanos menores, de tal forma que el último en nacer ve empeorada su situación al llegar a una familia de menor madurez y además no tener un hermano menor a quien enseñar.

Sin embargo, en otros estudios se destaca la posible existencia de otro efecto que va en dirección contraria. Por ejemplo, Behrman, Pollack y Taubman (1995) trabajan bajo un marco teórico completamente distinto, el modelo de roles, el cual postula que los hermanos no siempre serán un factor negativo en la educación de los otros, así un hermano con un buen desempeño escolar se puede transformar en un modelo a seguir por hermanos menores. En contraste, con la formulación de Becker (1960) esta hipótesis hace alusión al impacto positivo de la complementariedad de la calidad y la cantidad en la constelación de hermanos. Además, se enarbolan otros argumentos que también operan a favor de los hijos que nacen últimos, el hermano mayor eventualmente podría contribuir con su ingreso a los recursos familiares, de tal forma que el último hijo nacido podría obtener mayores niveles de educación ya que su familia tiene una restricción presupuestaría más flexible. Siguiendo en una línea argumental económica, Kessler (1991) puntualiza que en la medida en que los préstamos financieros individuales estén restringidos y los salarios tiendan a aumentar con la edad, los padres estarían mejor preparados para entregar recursos a 
los hijos que nacen más tardíamente. En el mismo sentido, se puede pensar que en la medida que los padres tienen más hijos van adquiriendo experiencia en el cuidado y educación de éstos ("learning by doing”), entregando una mejor niñez a los hijos que nacen últimos.

Un último ejemplo para graficar la diversidad de teorías que se pueden encontrar y los diversos sentidos que apoyan unas y otras es lo sugerido por Lindert (1977), quien señala que en los primeros años de vida, el hermano mayor no compite con otros niños por el tiempo y la atención de sus padres. A su vez, durante los últimos años de adolescencia, es el que nació último quien no necesita competir por el tiempo de los padres. El que nació al medio nunca puede disfrutar de este lujo.

Concluyendo, con respecto a los efectos del orden de nacimiento sobre los logros futuros existe una variada gama de argumentos que además no convergen. Por otra parte, la evidencia empírica está lejos de ser inequívoca. Por esto los resultados suelen interpretarse, no en el sentido de iluminar una regla general o posicionar una teoría sobre otra de manera universal, sino que simplemente como una descripción de una realidad social específica, la cual puede describirse en un momento dado de mejor manera bajo una u otra teoría.

Behrman y Taubman (1986) reportan un efecto significativo del orden de nacimiento, tanto sobre logros educacionales como sobre salario, el cual se da independiente de las preferencias de los padres y de los precios de la educación, y que favorece a los últimos en nacer. Kessler (1991) hace una crítica a este estudio planteando que el marco teórico que en él se encuentra versa en todo momento de efectos del orden de nacimiento pero siempre en términos relativos al número de hermanos y que el modelo planteado estima el efecto del orden de nacimiento en un sentido absoluto. Esto sesgaría la estimación del efecto del tamaño de la familia, ya que después de controlar por el orden de nacimiento absoluto, aún estaría omitido el orden de nacimiento relativo, el que debería estar correlacionado tanto con los logros como con el tamaño de la familia. Kessler (1991) no encuentra efectos significativos del orden de nacimiento sobre el salario ni sobre el crecimiento de estos. Wells (1995) hace un análisis de pares de hermanos incorporando también como variable el género de estos. Wells termina reportando evidencia que apoya la teoría del "tutor" y en consecuencia que el entorno familiar afecta diferente a los hijos según orden de nacimiento y género. Cheng (1998) con datos obtenidos en Taiwán, estudia los beneficios para un hijo que nace teniendo un hermano mayor bajo la hipótesis del "Role Model". Para mujeres encuentra un efecto positivo asociado a nacer último, en cambio, para hombres el efecto encontrado es inverso. Conley y Glauber (2005) en su reciente trabajo determinan que el efecto negativo del tamaño de la familia es uniformemente más fuerte para hijos nacidos más tardíamente que para los primerizos. Finalmente, Black, Devereux y Salvanes (2005) encuentran efectos significativos del orden de nacimiento en donde se ven favorecidos los primeros en nacer. El carácter mixto de la evidencia del orden de nacimiento sobre los logros plantea la necesidad de continuar con la investigación sobre el tema. 


\section{MODELO ECONOMETRICO}

\subsection{Modelo básico}

El enfoque econométrico que se sigue en esta investigación es el de Black, Devereux and Salvanes (2005). Se propone una función de producción educacional donde el producto son los años de educación:

$$
S_{i j}=\beta_{0}+\beta_{1} \operatorname{Size}_{j}+\beta_{2} B_{i j}+\beta_{3} X_{i j}+\beta_{4} W_{j}+\varepsilon_{i j}
$$

Donde $S_{i j}$ es los años de educación del individuo (hijo) i en la familia j, Size $e_{j}$ es el número de menores en la familia $\mathrm{j}, B_{i j}$ es el orden de nacimiento del menor i en la familia j, $X_{i j}$ es un vector de características del individuo como su edad, y $W_{j}$ es un vector de características de la familia que son iguales para todos los menores de la familia j. Entre estas, educación de la madre y del padre, edad de la madre y del padre, nivel socioeconómico del hogar durante la niñez del entrevistado.

\subsection{Usando gemelos}

Uno de los problemas econométricos que enfrenta esta ecuación es la endogeneidad del tamaño de la familia. La existencia de gemelos, sin embargo, aumenta el tamaño del hogar independientemente de las preferencias por este. Por lo tanto, se utilizan variables instrumentales para el tamaño del hogar. Los primeros en utilizar esta idea fueron Rosenzweig and Wolpin (1980). En ese modelo los padres tienen un número de niños óptimos, por lo tanto el nacimiento de gemelos altera esta decisión óptima exógenamente. El modelo es como sigue:

$$
\begin{aligned}
& S_{i j}=\beta_{0}+\beta_{1} \operatorname{Size}_{j}+\beta_{2} B_{i j}+\beta_{3} X_{i j}+\beta_{4} W_{j}+\varepsilon_{i j} \\
& \operatorname{Size}_{i j}=\gamma_{0}+\gamma_{1} \operatorname{Twin}_{i j}+\mu_{i j}
\end{aligned}
$$

Donde $\operatorname{Twin}_{i j}$ es una variable indicador que vale 1 si el individuo es gemelo en el nacimiento n-ésimo y 0 si no. Esta ecuación se estima para diferentes muestras según el número de nacimientos. Entonces, se toman en cuenta primero todos los hogares con dos y más nacimientos y se considera como instrumento la existencia de gemelos en el segundo nacimiento, después con tres y más nacimientos, y así sucesivamente. Además, se miden los efectos sobre los hijos nacidos hasta el nacimiento de gemelos. Estas restricciones permiten, por una parte, restringir la comparación a familias que en principio tenían preferencias en promedio iguales por tamaño de familia. Y por otra, enfrentar el problema de cambio en las preferencias por tamaño del hogar después de haber tenido gemelos. 


\subsection{Efectos fijos de familia}

Dada la disponibilidad de información podemos utilizar efectos fijos de familia en el caso del orden de nacimiento. Esto corrige por todas las características al interior del hogar que son iguales para todos los menores.

$$
S_{i j}=\delta_{0}+\delta_{1} B_{i j}+\delta_{2} F_{j}+\omega_{i j}
$$

\section{DATOS}

Los datos utilizados en esta investigación provienen de la Encuesta de Protección Social 2002. Esta se llevó a cabo entre junio de 2002 y enero de 2003 y fue realizada en conjunto por la Subsecretaría de Previsión Social del Gobierno de Chile y el Centro de Microdatos del Departamento de Economía de la Universidad de Chile. Se entrevistaron a 17.246 afiliados al sistema previsional del país.

La ventaja que ofrece esta encuesta radica en que reporta amplia información relevante sobre el entorno socioeconómico en que creció el individuo, y además, reporta información sobre los menores con los que convivió el entrevistado durante su infancia, poniendo a disposición no sólo la educación del entrevistado sino también la edad y educación de todos los hermanos de éste. Esto amplía la muestra a 77.320 personas incluyendo entrevistados y sus hermanos y hermanas. De estos hay variables como edad, educación, género, educación de los padres, edad de los padres y nivel socioeconómico durante la infancia. Información que nunca antes estuvo disponible en Chile y que permite la corrección por correlación al interior del hogar como la estimación con efectos fijos en el caso del orden de nacimiento.

En el caso de esta investigación se conservaron los datos de las familias en la cual todos los integrantes están vivos. Además, se consideraron a las familias cuya madre había nacido entre 1917 y 1957. Así, en el año 2002 no había ninguna madre menor de 45 años. Esto nos deja con una muestra de 27.294 personas, que pertenecen a 6.300 familias.

La Tabla 1 reporta información estadística respecto a la muestra utilizada en esta investigación. Los años de educación promedio de los entrevistados y sus hermanos son 10,6 años, con edad promedio de 33 años. Las madres tienen en promedio 59 años y los padres 63 años, con educación promedio de 6,3 y 6,9 años respectivamente. El orden de nacimiento promedio es 3 y el tamaño del hogar (número total de menores) es 5,5. Cuando el orden de nacimiento era mayor que 10 se agrupó en 10, y cuando la variable tamaño de familia era mayor a 12 se agrupó en 12 . Además, $5 \%$ de la muestra son niños nacidos en nacimientos múltiples, lo que equivale a 1.283 niños.

La Tabla 2 muestra los promedios de años de educación de la persona y sus padres, por tamaño de la familia y orden de nacimiento. Vemos que estos van monotónicamente disminuyendo a medida que el tamaño de la familia crece y a medida que el orden de nacimiento también crece. Una diferencia se puede observar en quienes pertenecen a una familia con un hijo único, en los cuales los promedios de años de educación de la madre y del padre son menores que en aquellos que tienen tamaño 2,3 y 4 . 
Esto coincide con la literatura internacional, los hijos únicos tienen desventajas en educación con el resto de la población.

TABLA 1

ESTADISTICA DESCRIPTIVA

\begin{tabular}{|lrrc|}
\hline & Media & D.E. & Obs. \\
\hline Educación & 10,60 & 3,58 & 27.294 \\
Edad & 33,17 & 10,01 & 27.294 \\
Edad Madre & 59,74 & 10,06 & 27.294 \\
Edad Padre & 63,63 & 10,64 & 27.294 \\
Educación Madre & 6,38 & 3,92 & 27.294 \\
Educación Padre & 6,95 & 4,22 & 27.294 \\
Orden de Nacimiento & 3,22 & 2,10 & 27.294 \\
Tamaño del Hogar & 5,57 & 2,61 & 27.294 \\
Pobre =1 & 0,40 & 0,49 & 27.294 \\
Gemelo = 1 & 0,05 & 0,21 & 27.294 \\
\hline
\end{tabular}

Nota: El número de observaciones es 27.294.

TABLA 2

EDUCACION Y EDUCACION DE LOS PADRES POR ORDEN DE NACIMIENTO

\begin{tabular}{|cccccc|}
\hline Tamaño del Hogar & Educación & $\begin{array}{c}\text { Educación } \\
\text { Madre }\end{array}$ & $\begin{array}{c}\text { Educación } \\
\text { Padre }\end{array}$ & Pobre $=1$ & Obs. \\
\hline 1 & 11,69 & 7,00 & 8,21 & 0,28 & 340 \\
2 & 12,19 & 8,50 & 8,92 & 0,26 & 1.866 \\
3 & 11,90 & 8,01 & 8,59 & 0,27 & 4.431 \\
4 & 11,28 & 7,41 & 7,95 & 0,32 & 4.597 \\
5 & 10,54 & 6,27 & 6,85 & 0,39 & 4.053 \\
6 & 10,23 & 5,75 & 6,41 & 0,44 & 3.275 \\
7 & 9,78 & 5,34 & 5,70 & 0,50 & 2.568 \\
8 & 9,42 & 5,14 & 5,58 & 0,49 & 2.016 \\
9 & 9,28 & 4,43 & 5,28 & 0,57 & 1.548 \\
10 & 9,30 & 4,48 & 5,01 & 0,54 & 1.101 \\
11 & 8,74 & 4,70 & 4,89 & 0,66 & 594 \\
12 & 8,81 & 3,71 & 4,88 & 0,61 & 905 \\
\hline Orden de Nacimiento & & & & & \\
\hline 1 & 11,27 & 7,02 & 7,60 & 0,35 & 6.366 \\
2 & 11,09 & 7,00 & 7,53 & 0,36 & 6.045 \\
4 & 10,60 & 6,73 & 7,28 & 0,37 & 5.083 \\
5 & 10,04 & 6,08 & 6,67 & 0,42 & 3.549 \\
6 & 9,84 & 5,49 & 6,09 & 0,47 & 2.356 \\
7 & 9,82 & 5,14 & 5,73 & 0,51 & 1.540 \\
8 & 9,70 & 4,82 & 5,33 & 0,54 & 987 \\
9 & 9,72 & 4,46 & 5,11 & 0,55 & 619 \\
10 & 9,79 & 4,14 & 4,88 & 0,59 & 372 \\
& 9,56 & 3,79 & 4,43 & 0,63 & 377 \\
\hline
\end{tabular}




\section{RESULTADOS}

En esta sección presentaremos los resultados usando los modelos econométricos descritos anteriormente. La Tabla 3 presenta los resultados del modelo básico. La variable tamaño de familia se incluye en forma continua y en forma discreta. El efecto del tamaño de la familia en la educación en este modelo es negativo y significativo. Incluso, después de controlar por orden de nacimiento, este efecto se incrementa, y es aún importante después de controlar por varias variables sociodemográficas. Estos son indicadores de edad, género, educación de madre y padre, edad de padre y madre.

$\mathrm{Al}$ considerar los efectos en forma discreta vemos que el efecto es no lineal. Las familias con 2 hermanos tienen significativamente más educación que las familias con hijo único. Las familias con 3 y 4 menores no tienen diferencias significativas con las de hijo único al controlar por variables sociodemográficas, por eso tienen menores resultados que familias de 2 hijos. Las familias de 5 y más menores sí tienen un efecto negativo sobre el rendimiento educacional.

Con respecto al orden de nacimiento, vemos que al contrario de lo encontrado por Black, Devereux y Salvanes (2005) el efecto del orden de nacimiento es no lineal y significativo. Es decir, los primeros en nacer y menores cuyo orden es 5 y más alcanzan mayores años de educación que los que nacen segundo a cuarto, incluso después de controlar por variables sociodemográficas y tamaño del hogar. Como se dijo antes los efectos encontrados en la literatura no tienen un signo único. En este caso, el resultado es por una parte explicado por el efecto que tiene el tiempo de exclusividad del menor que nace primero como por la experiencia de los padres en la crianza de los niños para los que nacen después. También, el efecto profesor en el sentido que los menores que nacen más temprano enseñan a quienes nacen más tarde. Por último y quizás más relevante es el efecto que puede tener el hecho de que hermanos mayores tengan que salir al mercado laboral antes para así mantener a los menores, alcanzando estos últimos mayores años de educación. Esto, sin embargo, no se cumple para el primero en nacer sino para quienes le siguen.

En el siguiente análisis se distinguen el efecto orden de nacimiento considerando familias de igual tamaño. Los resultados se muestran en la Tabla 4. Todas las regresiones incluyen como controles indicadores de edad, género, educación de madre y padre y edad de padre y madre. Al observar los efectos del orden de nacimiento sobre el logro educacional vemos que en familias de 2 y 3 hermanos este efecto es negativo y creciente, siendo menos favorecidos quienes nacen más tarde, sin embargo los parámetros no son significativos. En familias con 4 hermanos también el más favorecido es quien nace primero, aunque en este caso sí aparece un efecto más negativo y significativo en el tercero en nacer. Para familias con 5 y 6 hermanos ocurre algo similar, siendo menos favorecidos los terceros y cuartos en nacer. Algo diferente pasa en familias de 7 hermanos, donde los parámetros del orden de nacimiento son positivos, es decir, se ven más favorecidos los que nacen más tarde. En familias de 8 y más hermanos de nuevo aparece un efecto negativo de nacer en el medio, pero este se invierte pasando a favorecer a quienes nacen últimos. 


\section{TABLA 3}

EFECTOS DEL TAMAÑO DE FAMILIA Y EL ORDEN DE NACIMIENTO EN AÑOS DE EDUCACION: REGRESION OLS

\begin{tabular}{|c|c|c|c|c|c|c|}
\hline & (1) & $(2)$ & (3) & (4) & $(5)$ & (6) \\
\hline Tamaño de Familia & $\begin{array}{c}-0,383 * * * \\
(0,0170)\end{array}$ & & $\begin{array}{c}-0,415 \text { *** } \\
(0,0186)\end{array}$ & & $\begin{array}{c}-0,297 * * * \\
(0,0187)\end{array}$ & \\
\hline Tamaño de Familia $=2$ & & $\begin{array}{l}0,501 * * \\
(0,204)\end{array}$ & & $\begin{array}{l}0,570 * * * \\
(0,205)\end{array}$ & & $\begin{array}{l}0,506^{* * * *} \\
(0,184)\end{array}$ \\
\hline Tamaño de Familia $=3$ & & $\begin{array}{c}0,213 \\
(0,198)\end{array}$ & & $\begin{array}{l}0,398 * * \\
(0,199)\end{array}$ & & $\begin{array}{l}0,421^{* *} \\
(0,180)\end{array}$ \\
\hline Tamaño de Familia = 4 & & $\begin{array}{l}-0,413^{* *} \\
(0,200)\end{array}$ & & $\begin{array}{c}-0,148 \\
(0,202)\end{array}$ & & $\begin{array}{c}0,130 \\
(0,182)\end{array}$ \\
\hline Tamaño de Familia $=5$ & & $\begin{array}{l}-1,148^{* * * *} \\
(0,206)\end{array}$ & & $\begin{array}{l}-0,873 * * * \\
(0,208)\end{array}$ & & $\begin{array}{c}-0,317^{*} \\
(0,189)\end{array}$ \\
\hline Tamaño de Familia $=6$ & & $\begin{array}{l}-1,464 \text { *** } \\
(0,213)\end{array}$ & & $\begin{array}{c}-1,233 * * * \\
(0,216)\end{array}$ & & $\begin{array}{c}-0,698^{* * * *} \\
(0,200)\end{array}$ \\
\hline Tamaño de Familia $=7$ & & $\begin{array}{l}-1,907 * * * \\
(0,230)\end{array}$ & & $\begin{array}{l}-1,740 * * * \\
(0,235)\end{array}$ & & $\begin{array}{l}-1,111 \text { *** } \\
(0,215)\end{array}$ \\
\hline Tamaño de Familia = 8 & & $\begin{array}{l}-2,274 * * * \\
(0,255)\end{array}$ & & $\begin{array}{l}-2,186 * * * \\
(0,260)\end{array}$ & & $\begin{array}{l}-1,423 \text { *** } \\
(0,230)\end{array}$ \\
\hline Tamaño de Familia $=9$ & & $\begin{array}{l}-2,416 * * * \\
(0,278)\end{array}$ & & $\begin{array}{l}-2,416^{* * * *} \\
(0,287)\end{array}$ & & $\begin{array}{l}-1,665^{* * *} \\
(0,256)\end{array}$ \\
\hline Tamaño de Familia $=10$ & & $\begin{array}{l}-2,390^{* * * *} \\
(0,324)\end{array}$ & & $\begin{array}{l}-2,472 * * * \\
(0,331)\end{array}$ & & $\begin{array}{l}-1,655 * * * \\
(0,300)\end{array}$ \\
\hline Tamaño de Familia = 11 & & $\begin{array}{l}-2,952 * * * \\
(0,359)\end{array}$ & & $\begin{array}{c}-3,086 * * * \\
(0,367)\end{array}$ & & $\begin{array}{l}-2,141 * * * \\
(0,320)\end{array}$ \\
\hline Tamaño de Familia $>=12$ & & $\begin{array}{l}-2,885^{* * *} \\
(0,363)\end{array}$ & & $\begin{array}{l}-3,111 * * * * \\
(0,377)\end{array}$ & & $\begin{array}{l}-2,041 * * * \\
(0,337)\end{array}$ \\
\hline Orden de Nacimiento $=2$ & & & $\begin{array}{l}-0,0857 \text { ** } \\
(0,0423)\end{array}$ & $\begin{array}{l}-0,140 * * * \\
(0,0421)\end{array}$ & $\begin{array}{c}-0,103 * * \\
(0,0442)\end{array}$ & $\begin{array}{c}-0,149 * * * \\
(0,0443)\end{array}$ \\
\hline Orden de Nacimiento $=3$ & & & $\begin{array}{c}-0,370 * * * \\
(0,0510)\end{array}$ & $\begin{array}{c}-0,425 * * * \\
(0,0497)\end{array}$ & $\begin{array}{c}-0,241 * * * \\
(0,0586)\end{array}$ & $\begin{array}{c}-0,302^{* * *} * \\
(0,0582)\end{array}$ \\
\hline Orden de Nacimiento $=4$ & & & $\begin{array}{c}-0,558 * * * * \\
(0,0668)\end{array}$ & $\begin{array}{c}-0,524 * * * \\
(0,0630)\end{array}$ & $\begin{array}{c}-0,215^{* * *} \\
(0,0766)\end{array}$ & $\begin{array}{c}-0,235 * * * \\
(0,0756)\end{array}$ \\
\hline Orden de Nacimiento $=5$ & & & $\begin{array}{c}-0,391 * * * \\
(0,0776)\end{array}$ & $\begin{array}{c}-0,283^{* * *} * \\
(0,0723)\end{array}$ & $\begin{array}{l}-0,103 \\
(0,0944)\end{array}$ & $\begin{array}{c}-0,0606 \\
(0,0931)\end{array}$ \\
\hline Orden de Nacimiento $=6$ & & & $\begin{array}{c}-0,0343 \\
(0,0906)\end{array}$ & $\begin{array}{c}0,0450 \\
(0,0855)\end{array}$ & $\begin{array}{c}0,0988 \\
(0,117)\end{array}$ & $\begin{array}{c}0,171 \\
(0,114)\end{array}$ \\
\hline Orden de Nacimiento $=7$ & & & $\begin{array}{l}0,207 * * \\
(0,104)\end{array}$ & $\begin{array}{l}0,260 * * * \\
(0,100)\end{array}$ & $\begin{array}{l}0,278^{* *} \\
(0,140)\end{array}$ & $\begin{array}{l}0,349 * * \\
(0,137)\end{array}$ \\
\hline Orden de Nacimiento $=8$ & & & $\begin{array}{l}0,583 * * * \\
(0,129)\end{array}$ & $\begin{array}{l}0,537 * * * \\
(0,127)\end{array}$ & $\begin{array}{l}0,560 * * * \\
(0,165)\end{array}$ & $\begin{array}{l}0,570 \text { *** } \\
(0,163)\end{array}$ \\
\hline Orden de Nacimiento $=9$ & & & $\begin{array}{l}0,970 * * * \\
(0,159)\end{array}$ & $\begin{array}{l}0,780 * * * \\
(0,155)\end{array}$ & $\begin{array}{l}0,732 * * * \\
(0,197)\end{array}$ & $\begin{array}{l}0,652 \text { *** } \\
(0,194)\end{array}$ \\
\hline Orden de Nacimiento $=10$ & & & $\begin{array}{l}1,226 * * * \\
(0,219)\end{array}$ & $\begin{array}{l}0,844 * * * \\
(0,196)\end{array}$ & $\begin{array}{l}0,979 * * * \\
(0,244)\end{array}$ & $\begin{array}{l}0,730 * * * \\
(0,231)\end{array}$ \\
\hline Constante & $\begin{array}{c}12,74 * * * \\
(0,0861)\end{array}$ & $\begin{array}{l}11,69 \text { *** } \\
(0,185)\end{array}$ & $\begin{array}{c}13,06 * * * \\
(0,0866)\end{array}$ & $\begin{array}{l}11,69 * * * \\
(0,185)\end{array}$ & $\begin{array}{l}-3,380 * * * \\
(0,913)\end{array}$ & $\begin{array}{l}-4,702 \text { *** } \\
(0,942)\end{array}$ \\
\hline Observaciones & 27.294 & 27.294 & 27.294 & 27.294 & 27.294 & 27.294 \\
\hline R-squared & 0,078 & 0,084 & 0,086 & 0,089 & 0,373 & 0,375 \\
\hline
\end{tabular}

Errores Estándares Robustos entre Paréntesis. $* * * \mathrm{p}<0,01, * * \mathrm{p}<0,05, * \mathrm{p}<0,1$.

Nota: Columnas (5) y (6) incluyen como controles demográficos indicadores de: edad, género, educación de la madre, educación del padre y edad del padre y de la madre. 


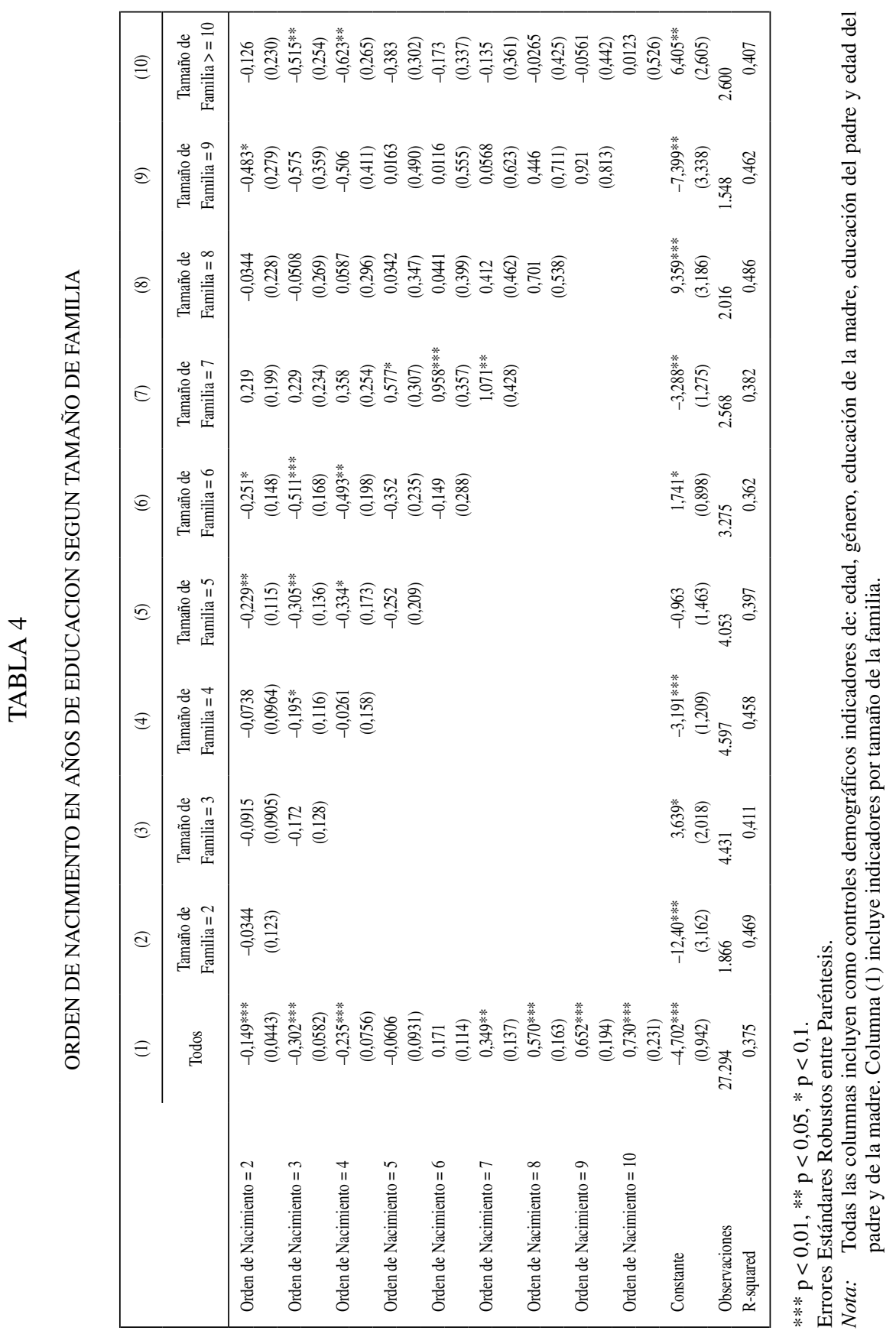




\section{Usando gemelos como variable instrumental para fertilidad}

Ahora se utiliza la existencia de gemelos como variable instrumental para el tamaño de familia. La idea detrás de esto es que las familias pueden elegir tener un hijo, sin embargo, no pueden elegir tener gemelos. Estos se distribuyen exógenamente. Al igual que en Black, Devereux y Salvanes (2005) el 5\% de la muestra son gemelos. La Tabla 5 muestra la frecuencia de gemelos por orden de nacimiento. Estos son más frecuentes en los primeros y segundos órdenes, y para cada grupo de orden de nacimiento son aproximadamente el 5\% de la muestra.

\section{TABLA 5}

FRECUENCIA DE GEMELOS POR ORDEN DE NACIMIENTO

\begin{tabular}{|ccr|}
\hline $\begin{array}{c}\text { Número de } \\
\text { Nacimientos }\end{array}$ & Gemelos & Total \\
\hline 1 & $6,85 \%$ & 365 \\
2 & $5,36 \%$ & 1.998 \\
3 & $4,29 \%$ & 4.683 \\
4 & $3,75 \%$ & 4.666 \\
5 & $4,67 \%$ & 4.135 \\
6 & $4,40 \%$ & 3.272 \\
7 & $5,16 \%$ & 2.576 \\
8 & $5,35 \%$ & 1.944 \\
9 & $5,06 \%$ & 1.482 \\
10 y más & $5,80 \%$ & 2.173 \\
\hline Total & $4,70 \%$ & 27.294 \\
\hline
\end{tabular}

Para que las estimaciones de variables instrumentales sean consistentes, los instrumentos deben estar no correlacionados con el término de error en la ecuación principal. Esto es, no debe ser una variable omitida relevante para la ecuación de educación. Una forma de que esto sea así es que la existencia de gemelos esté correlacionada con alguna variable no observada del hogar o el individuo. Como forma de chequear que el instrumento es válido se estimó un modelo de probabilidad lineal donde la variable dependiente es una variable indicador si el individuo es gemelo y 0 si no, y las variables explicativas son la educación de madre y padres. La Tabla 6 muestra los resultados. Como podemos notar, ambas variables son no significativas. Por otra parte, una variable que sí está correlacionada con ser gemelo es la edad de la madre al nacer, la cual mientras mayor es mayor la probabilidad. ${ }^{1}$ Para eso incluimos los indicadores de año de nacimiento de la madre y del individuo.

\footnotetext{
1 Jacobsen, Pearce and Rosenbloom (1999) reportan este resultado, entre otros.
} 
TABLA 6

TESTEANDO EXOGENEIDAD DE GEMELOS: MODELO DE PROBABILIDAD LINEAL

\begin{tabular}{|lccc|}
\hline & $(1)$ & $(2)$ & $(3)$ \\
\cline { 2 - 4 } & Gemelo $=1$ & Gemelo $=1$ & Gemelo $=1$ \\
\hline \multirow{2}{*}{ Educación de la Madre } & & & \\
& $-0,000235$ & & $-0,000545$ \\
Educación del Padre & $(0,000333)$ & & $(0,000443)$ \\
& & 0,000111 & 0,000441 \\
Constante & & $(0,000303)$ & $(0,000403)$ \\
& $0,0485^{* * *}$ & $0,0462^{* * *}$ & $0,0474 * * *$ \\
Observaciones & $(0,00250)$ & $(0,00245)$ & $(0,00265)$ \\
R-squared & 27.294 & 27.294 & 27.294 \\
& 0,000 & 0,000 & 0,000 \\
\hline
\end{tabular}

Errores Estándares Robustos entre Paréntesis.

$* * * \mathrm{p}<0,01, * * \mathrm{p}<0,05, * \mathrm{p}<0,1$.

A continuación se realizan las estimaciones usando el modelo de variables instrumentales explicado en la sección anterior. Cabe recordar que se tomaron diferentes grupos dependiendo del momento en el cual habían nacido gemelos. Los efectos se midieron sobre los niños nacidos hasta el nacimiento de los gemelos. Por ejemplo, si los gemelos nacieron en el segundo nacimiento, se consideró a las familias de dos y más nacimientos, y el efecto se midió sobre los hijos que nacieron primeros y segundos. Estas dos estrategias se usan para reducir un sesgo producido por cambios en las preferencias por tamaño del hogar después del nacimiento de gemelos. La Tabla 7 muestra los resultados. Todas las regresiones incluyen indicadores de género, edad y edad y educación de padre y madre. Se reportan los resultados OLS y IV. Lo primero que podemos destacar es que los instrumentos son siempre altamente significativos. En segundo lugar, el efecto del tamaño de familia sobre el logro educacional es también negativo y siempre significativo reflejando que existe un trade-off entre cantidad y calidad que va creciendo con el número de nacimientos. Por otra parte, los efectos del orden de nacimiento aparecen de nuevo beneficiando al hijo mayor y se repite el efecto no lineal encontrado en las regresiones anteriores.

\section{Modelo de efectos fijos de familia}

A continuación presentamos los resultados al considerar un modelo de efectos fijos de familia. En este caso solamente podemos evaluar los resultados de orden de nacimiento. El efecto fijo tomará en cuenta el efecto del tamaño de familia, y todas las características del hogar comunes a todos los hermanos. La Tabla 8 muestra los resultados. A diferencia de los resultados sin efectos fijos mostrados en la Tabla 2, la gran mayoría de los parámetros tiene signo negativo, o sea efectos 


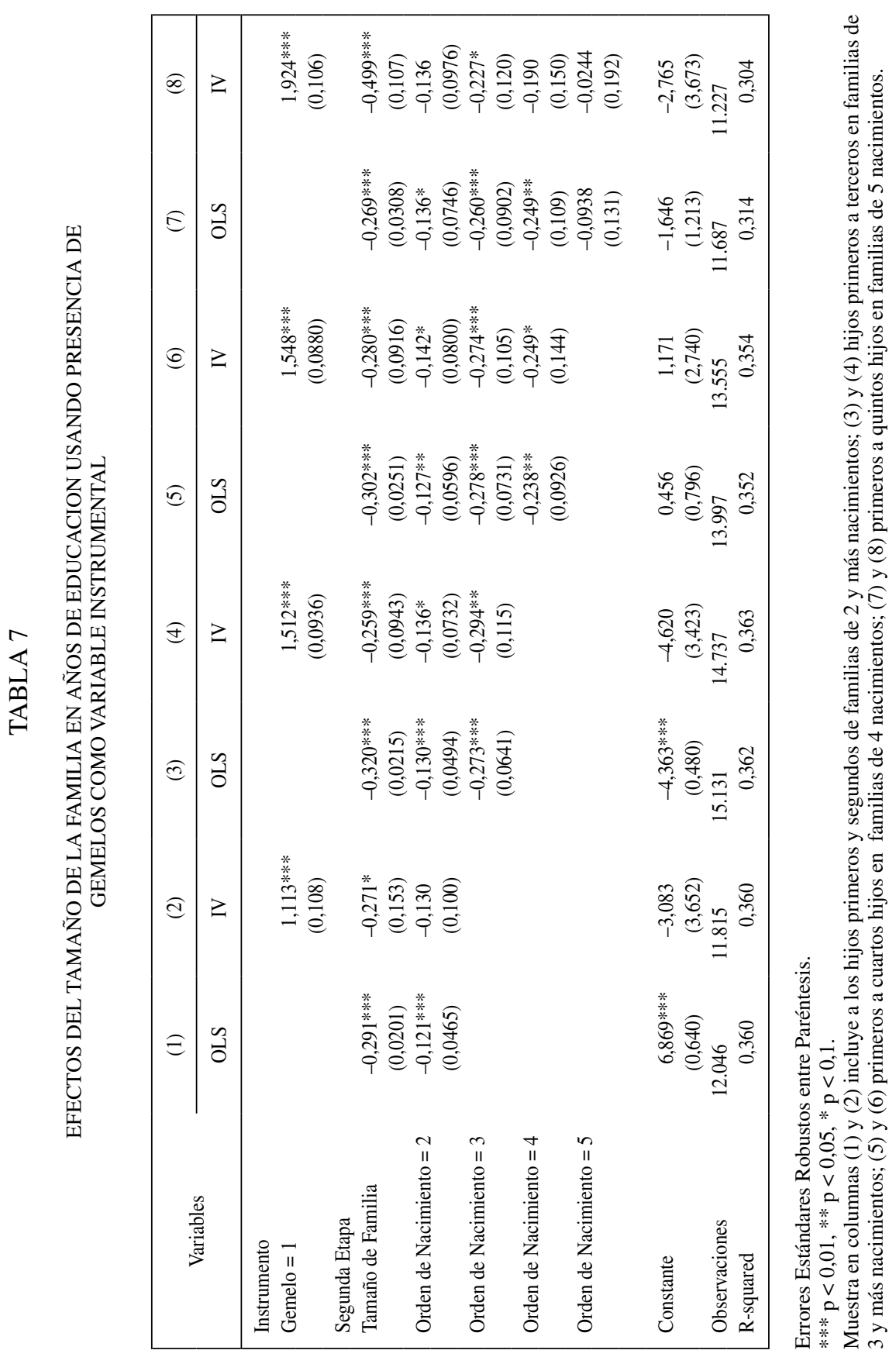




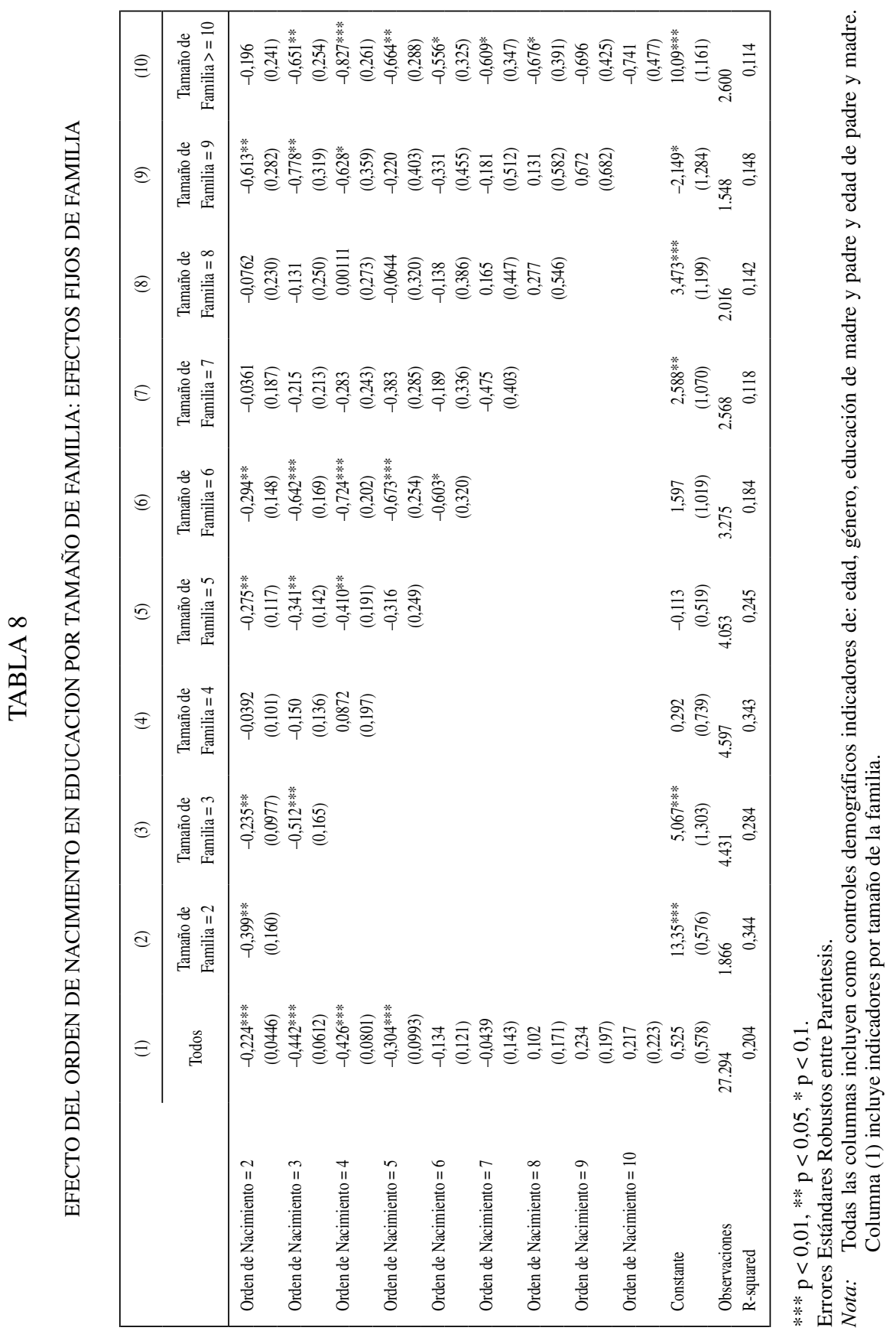


negativos de nacer con posterioridad, aunque aún persisten con signo positivo los últimos en nacer en familias de tamaño cuatro, ocho y nueve. Al tomar en cuenta la significancia de los parámetros vemos que al igual que en Tabla 2 existiría en algunos casos un efecto no lineal. Lo que no hay duda es que los hijos mayores son los más favorecidos.

\section{Efectos heterogéneos}

Por último, en esta sección investigamos posibles efectos heterogéneos por género, edad de la madre, educación de la madre y condición socioeconómica durante la niñez. La Tabla 9 muestra los resultados.

En primer lugar, diferenciamos las muestras por sexo del niño. Los resultados OLS muestran que los efectos negativos del tamaño del hogar son mayores en magnitud para los niños que para las niñas. Las estimaciones de variables instrumentales acentúan estas diferencias por género cuando se usa como instrumento el nacimiento de gemelos en segundo o tercer orden. Sin embargo, cuando usamos gemelos en el cuarto orden los efectos negativos del tamaño del hogar reaparecen tanto para niñas como para niños. Hay que recordar que estos efectos son sobre los niños nacidos hasta el nacimiento de los gemelos, inclusive. Por otra parte, los efectos del orden de nacimiento son más negativos para las niñas que para los niños. Esto quiere decir que se pierde más en nacer con posterioridad si el pequeño es niña que si es niño.

También se segmentó la muestra por nivel educacional de la madre. Ya que es una muestra de mujeres mayores y los niveles educacionales antes eran más bajos, dividimos la muestra entre educación básica completa y menos, y más que educación básica completa. Podemos notar que en las estimaciones OLS los efectos son más negativos para niños cuya madre tiene mayor educación lo que va en sentido contrario al efecto de las restricciones de ingreso. A su vez, los resultados de variables instrumentales confirman este resultado cuando se usa como instrumento gemelos nacidos en segundo y tercer orden, y lo contradicen al usar como instrumento gemelos en el cuarto orden. En este último caso, el efecto más negativo es para los hogares donde la madre es menos educada. Por otra parte, los resultados de orden de nacimiento muestran efectos más negativos en aquellos que nacen más tarde y tienen una madre con menor educación.

Otra variable de interés es la edad de nacimiento de la madre. Por un tema de número de observaciones dividimos la muestra entre madres que nacieron antes de 1942 y madres que nacieron después de 1942. Cohortes más jóvenes tuvieron acceso a mecanismos de control de natalidad. Los efectos del tamaño de la familia son aún negativos para ambos cohortes. Al corregir por orden de nacimiento el efecto negativo es menor en hogares cuya madre es más joven. Los estimadores de variables instrumentales básicamente confirman este resultado. Los efectos del orden de nacimiento negativo para los hermanos del medio son mayores en familias cuya madre es también más joven. 


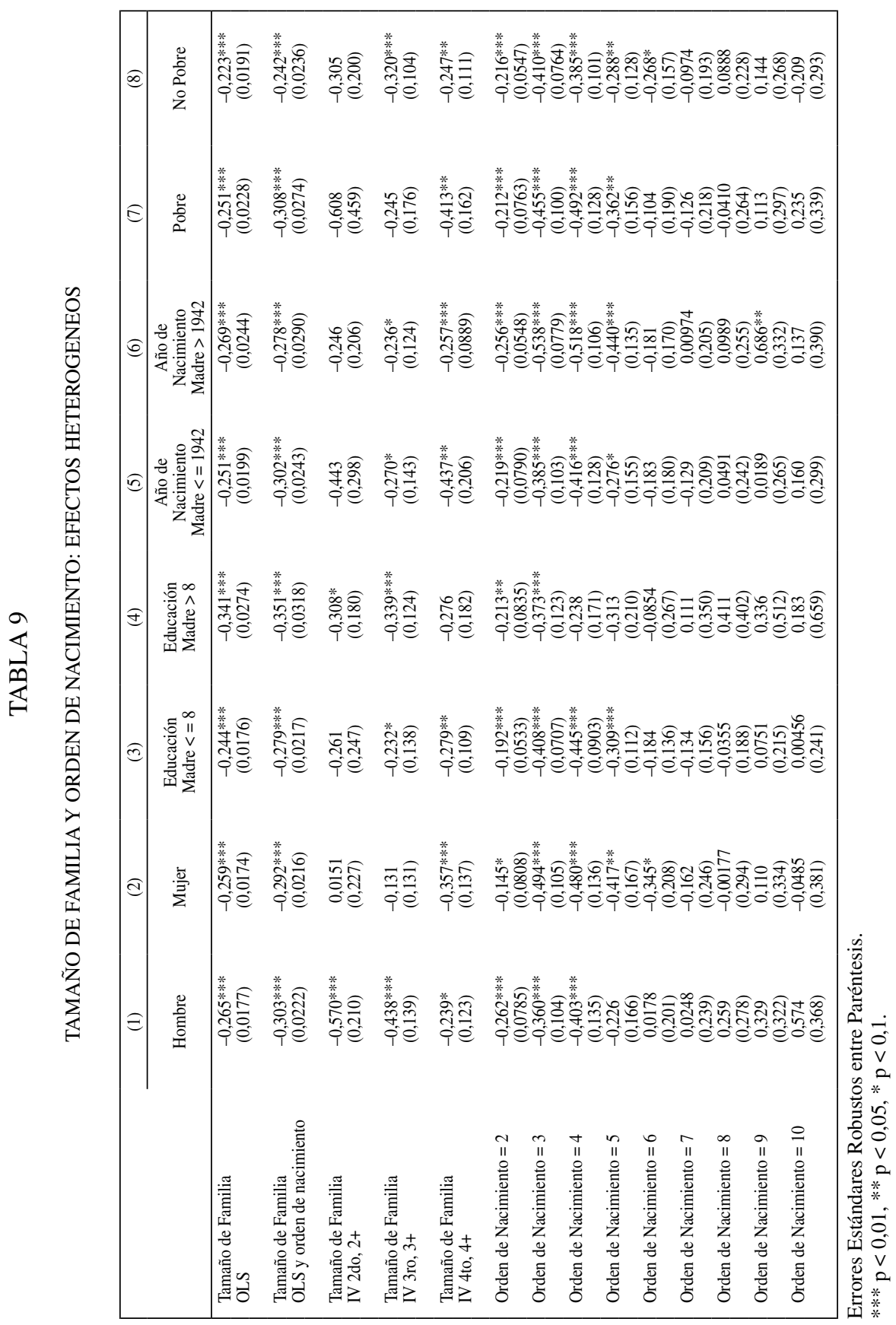


Finalmente, diferenciamos las muestras entre aquellas familias que declaran haber vivido en pobreza o extrema pobreza durante la niñez y aquellas que no vivían bajo esa condición socioeconómica. Vemos que el efecto negativo del tamaño del hogar y el orden de nacimiento es mayor en familias más pobres. Aunque aún es negativo y significativo en familias no pobres. Cuando usamos como instrumento para el tamaño de la familia el nacimiento de gemelos en segundo lugar no tenemos efectos del tamaño de la familia independiente del nivel socioeconómico. Cuando utilizamos como instrumento el nacimiento de gemelos en tercer orden encontramos el efecto en familias no pobres. Y cuando utilizamos como instrumento el nacimiento de gemelos en cuarto lugar tenemos efectos negativos del tamaño de la familia para ambos grupos socioeconómicos. Finalmente, los efectos del orden de nacimiento sugieren un efecto en perjuicio de los hermanos del medio mayor para los que vivieron en pobreza.

\section{CONCLUSIONES}

El principal resultado de esta investigación es la existencia de un efecto causal significativamente negativo del tamaño de la familia sobre los logros futuros de los individuos. Hallazgo que es consistente con la gran mayoría de la evidencia internacional al respecto, respaldando la preponderancia en Chile de un efecto de dilución de recursos al interior de la familia en la medida que ésta crece, lo que resulta en menores logros de los individuos que pertenecen a ellas.

La existencia de un trade-off entre calidad y cantidad de los hijos pone de manifiesto la relevancia de una planificación familiar apropiada que permita la adecuada asignación de recursos cuya dotación está relativamente fija al interior de la familia y que se destinan a mejorar las capacidades de generación de logros en el futuro de los hijos. Sin embargo, la existencia de un efecto negativo y significativo asociado a los hijos únicos hace pensar en un cierto grado de complementariedad entre hermanos que existe más allá de la competencia por recursos, si bien existe esta relación negativa entre "tamaño de la familia" y "logros", el óptimo no está en familias con un solo hijo.

Con respecto a los efectos del orden de nacimiento este muestra ser negativo para los hermanos nacidos inmediatamente posteriores al hermano mayor. Sin embargo, este efecto se hace no significativo y en ocasiones positivo si vemos los efectos sobre los menores en familias de mayor cantidad de hermanos. Estos resultados tienen una mayor concordancia con las teorías que indican que el tiempo es escaso. Los hijos mayores disfrutan de alguna exclusividad del tiempo con sus padres y eso muestra ser significativo en este análisis. Por otra parte, el efecto no lineal es bastante permanente en nuestros análisis mostrando que podría existir un efecto profesor en el cual niños menores aprenden de sus hermanos mayores y alcanzan mayores niveles educacionales. 


\section{REFERENCIAS}

BAUER, T. and I. GANG (2001) "Sibling Rivalry in Educational Attainment: The German Case", Labor 15 (2), pp. 191-341.

BECKER, G. (1960). "An Economic Analysis of Fertility". En G. BECKER, J. DUESENBERRY and B. OKUN, Demographic and Economic Change in Developed Countries, Princeton University Press, NJ, pp. 225-256.

BECKER, G. y H. LEWIS (1973). "On the Interaction between the Quantity and Quality of Children", Journal of Political Economy 81 (2), pp. 279-288.

BECKER, G. y N. TOMES (1976). "Child Endowments and the Quantity and Quality of Children", Journal of Political Economy 84 (4), pp. 143-162.

BEHRMAN, J. y P. TAUBMAN (1986). "Birth Order, Schooling and Earnings", Journal of Labor Economics 4 (3), pp. 121-145.

BEHRMAN, J.; R. POLLACK y P. TAUBMAN (1995). From Parents to Child: Intrahousehold Allocations and Intergenerational Relations in the United States, University of Chicago Press, Chicago, USA.

BLACK, S.; P. DEVEREUX y K. SALVANES (2005). "The More the Merrier? The Effect of Family Size and Birth Order on Children's Education”, The Quarterly Journal of Economics 120 (2), pp. 669-700.

CHEN, H. (1998). "Empirical Studies of Human Capital Formation: The Role of Family, Sibling, and Neighborhood", PhD Dissertation, Virginia Polytechnic Institute and State University.

CONLEY, D. y R. GLAUBER (2005). "Parental educational investment and children's academic risk: Estimates of the impact of sibship size and birth order from exogenous variation in fertility", Working Paper 11302, NBER, April.

GUO, G. y L. VAN WEY (1999). "Sibship Size And Intellectual Development: Is the Relationship Causal?", American Sociological Review 64, pp. 169-187.

JACOBSEN, J.; J. WISHART PEARCE III and J. ROSENBLOOM (1999). "The Effects of Childbearing on Married Women's Labor Supply and Earnings: Using Twin Births as a Natural Experiment", Journal of Human Resources 3, pp. 449-474.

KESSLER, D. (1991). "Birth Order, Familie Size and Achievement: Family Structure and Wage Determination", Journal of Labor Economics 9 (4), pp. 413-426.

LINDERT, P. (1977). "Sibling Position and Achievement", Journal of Human Resources 12 (2), pp. 198-219.

ROSENZWEIG, M. y K. WOLPIN (1980). "Testing the Quantity-Quality Fertility Model: The Use of Twins as a Natural Experiment", Econometrica 48 (1), pp. 227-240.

STEELMAN, L.; B. POWELL, R. WERUM y S. CARTER (2002). "Reconsidering the Effects of Sibling Configuration: Recent Advances and Challenges", Annual Review of Sociology 28, pp. 243-269.

WELLS, T. (1995). "Does Family Background Effect Educational Attainment Differently According to Family Structure, Birth Order, and Sex?", National Survey of Families and Households, Working Paper $\mathrm{N}^{\circ} 70$.

ZANJONC, R. (1976). "Family Configuration and Intelligence", Science 192, No 4236, pp. 227-236. 